\title{
Polymorphisms of Vitamin D Signaling Pathway Genes and Calcium-Sensing Receptor Gene in respect to Survival of Hemodialysis Patients: A Prospective Observational Study
}

\author{
Alicja E. Grzegorzewska, ${ }^{1}$ Monika K. Świderska, ${ }^{2}$ Adrianna Mostowska, ${ }^{3}$ \\ Wojciech Warchol, ${ }^{4}$ and Paweł P. Jagodziński ${ }^{3}$ \\ ${ }^{1}$ Chair and Department of Nephrology, Transplantology and Internal Diseases, Poznan University of Medical Sciences, \\ Przybyszewskiego 49, 60-355 Poznań, Poland \\ ${ }^{2}$ Student Nephrology Research Group, Chair and Department of Nephrology, Transplantology and Internal Diseases, \\ Poznan University of Medical Sciences, Przybyszewskiego 49, 60-355 Poznań, Poland \\ ${ }^{3}$ Chair and Department of Biochemistry and Molecular Biology, Poznan University of Medical Sciences, Święcickiego 6, \\ 60-781 Poznań, Poland \\ ${ }^{4}$ Chair and Department of Biophysics, Poznan University of Medical Sciences, Grunwaldzka 6, 60-780 Poznań, Poland
}

Correspondence should be addressed to Alicja E. Grzegorzewska; alicja_grzegorzewska@yahoo.com

Received 27 April 2016; Revised 27 June 2016; Accepted 23 July 2016

Academic Editor: Xiangbing Wang

Copyright (c) 2016 Alicja E. Grzegorzewska et al. This is an open access article distributed under the Creative Commons Attribution License, which permits unrestricted use, distribution, and reproduction in any medium, provided the original work is properly cited.

\begin{abstract}
We evaluated in the 7-year prospective study whether variants in vitamin D pathway genes and calcium-sensing receptor gene (CASR) are determinants of mortality in hemodialysis (HD) patients $(n=532)$. HRM analysis was used for GC rs 2298849 , GC rs1155563, RXRA rs10776909, RXRA rs10881578, and CASR rs7652589 genotyping. GC rs7041, RXRA rs749759, VDR rs2228570, and $V D R$ rs 1544410 were genotyped using PCR-RFLP analysis. The minor allele in GC rs2298849 was associated with all-cause mortality in univariate analysis (HR 1.330, 95\% CI 1.046-1.692, $P=0.020$ ). Bearers of the minor allele in $G C$ rs 2298849 demonstrated higher infection/neoplasm mortality than major allele homozygotes also in multivariate analysis (HR 2.116, 95\% CI 1.096-4.087, $P=0.026$ ). Cardiovascular mortality was associated with major homozygosity (CC) in VDR rs 2228570 (HR $1.896,95 \%$ CI 1.163-3.091, $P=0.010)$. CC genotype patients were more often dyslipidemic than TT genotype subjects $(46.1 \%$ versus $31.9 \%$, $P=0.047)$. Dyslipidemics showed higher frequency of rs1544410_rs2228570 haplotype AC than nondyslipidemics (26 versus $18 \%$, $\left.P_{\text {corr }}=0.005\right)$, whereas TT genotype patients were at lower risk of dyslipidemia compared with CC/CT genotype patients (HR 0.59, 95\% CI 0.37-0.96, $P=0.04$ ). In conclusion, GC rs2298849 and VDR rs2228570 SNPs are associated with survival on HD. VDR-related cardiovascular mortality may occur due to connections of rs 2228570 with dyslipidemia.
\end{abstract}

\section{Introduction}

Patients who undergo renal replacement therapy (RRT) due to end-stage renal disease (ESRD) have higher prevalence of comorbidities and increased adjusted mortality risk compared with healthy population [1]. Cardiovascular diseases, infections, and cancers are the most common causes of death in this group of patients $[1,2]$.

Vitamin D (VD) deficiency is a mortality risk factor in hemodialysis (HD) patients [3, 4]. Associations between low VD status and neoplasms [5-7], diabetes mellitus [8,9], cardiovascular disease [10], myocardial infarction [11], bone fractures [12], and others have been discovered in recent years. What is more, VD is an important immunoregulator [13] and might play a key role in susceptibility to autoimmune disorders [13-15]. Lower plasma concentrations of VD binding protein were also associated with increased mortality risk in a 4-year prospective follow-up of nondiabetic HD patients [16].

Polymorphisms of VD signaling pathway genes influencing VD status and proper VD utilization include groupspecific component protein (VD binding protein) gene (GC), 
VD receptor gene (VDR), and downstream mediator of VD signaling, retinoid $\mathrm{X}$ receptor alpha gene (RXRA) [17-19]. Calcium-sensing receptor, encoded by specific gene (CASR), controls calcium homeostasis along with VD through the regulation of parathyroid hormone (PTH) production [20]. In secondary hyperparathyroidism, there is downregulation of vitamin D receptor, calcium-sensing receptor, and retinoid $\mathrm{X}$ receptor in parathyroid cells [21].

To date, few studies have investigated the effects of VD signaling pathway gene polymorphisms on survival of ESRD patients. Marco et al. [22] have found that BsmI polymorphism of $V D R$ influenced survival of $H D$ patients in a 4-year prospective study. However, VDR polymorphisms (BsmI and FokI) showed no impact on ninety-day survival rate of acute kidney injury patients [23]. GC, $V D R$, and $R X R A$ polymorphisms were not associated with risk of death in patients on RRT in our retrospective analysis [2]. CASR polymorphic variants had no impact on all-cause and cardiovascular mortalities in HD patients as well as on the occurrence of cardiovascular events and all-cause mortality in renal transplant recipients in retrospective studies [24, 25]. As retrospective analyses have drawbacks, including biases in the selection of patients, long-term prospective studies may yield more promising results in evaluation of associations between mentioned polymorphisms and survival of $\mathrm{HD}$ patients, as seems to be confirmed by the Marco et al. study [22].

The aim of this 7-year prospective study was to evaluate whether single nucleotide polymorphisms (SNPs) of vitamin $\mathrm{D}$ signaling pathway genes and CASR are determinants of survival in HD patients.

\section{Material and Methods}

2.1. Enrolment of Patients. HD patients living in the Greater Poland District, Poland, were enrolled into the prospective, observational study in January, 2009. Known status in respect to hepatitis B virus (HBV) susceptibility or infection was an inclusion criterion for our study, because the ability to produce antibodies to HBV surface antigen (anti-HBs) was one of parameters investigated as a predictor of survival. Therefore, the ability to produce anti-HBs antibodies had to be known at the start of the study. An exclusion criterion was renal transplantation prior to enrolment. Patients were in stable clinical condition for at least one month prior to enrolment. Characteristics of enrolled patients $(n=532)$ are shown in Table 1.

Coronary artery disease (CAD) was diagnosed based on a medical history, electrocardiograms, exercise stress test, and, in some cases, coronary angiography or computed tomography. Dyslipidemia was diagnosed according to the recommendations of the National Kidney Foundation/Kidney Disease Outcomes Quality Initiative (KDOQI) clinical practice guidelines [26].

2.2. Follow-Up. Patients were followed from January 30, 2009, to January 30, 2016. Results concerning 6-year survival (to January 30, 2015) of the same group of patients in respect to their ability to produce anti-HBs antibodies are presented in our earlier study [27].

The immediate cause of death was reported by a physician who declared death basing on medical evidence prior to death. Patients were mainly dying at hospital, and a nephrologist responsible for dialysis was usually arranging a patient hospitalization. Deaths that occurred outside medical facilities (at home, during outdoor activities) were declared by first aid physicians. Medical records of a deceased patient were available in each such case. The causes of death were recorded and categorized as cardiac (reported as myocardial infarction, sudden cardiac death, severe arrhythmias, cardiomyopathies, or cardiac failure), vascular (reported as cerebrovascular events, cerebral stroke, or generalized atherosclerosis), infectious (reported as sepsis, pneumonia, limb necrosis, pyonephrosis, or acute abdomen with peritonitis), neoplasms, or other/unknown.

Characteristics of HD patients at the end of a 7-year prospective study are shown in Table 2 .

2.3. Genotyping. Genotyping of vitamin $\mathrm{D}$ signaling pathway genes (VDR rs2228570, VDR rs1544410, RXRA rs10776909, RXRA rs10881578, RXRA rs749759, GC rs2298849, GC rs7041, and $G C$ rs1155563) was performed as previously described [2, 24, 28-31].

In brief, genomic DNA for a genotype analysis was isolated from peripheral blood lymphocytes by a salt-out extraction procedure. High-resolution melting curve (HRM) analysis was used for GC rs2298849, GC rs1155563, RXRA rs10776909, RXRA rs10881578, and CASR rs7652589 genotyping. GC rs7041, RXRA rs749759, VDR rs2228570, and $V D R$ rs1544410 were genotyped using the polymerase chain reaction-restriction fragment length polymorphism (PCRRFLP) analysis. The characteristics of analyzed polymorphisms are described in Supplementary Table 1 in Supplementary Material available online at http://dx.doi.org/ $10.1155 / 2016 / 2383216$. Primer sequences and conditions for PCR-RFLP and HRM analyses are presented in Supplementary Table 2. Approximately $10 \%$ of the randomly chosen samples were regenotyped. Samples that failed the genotyping were excluded from further statistical analyses.

Genotyping of tested SNPs was performed in groups of 435-472 patients (Table 3).

2.4. Statistical Methods. The results are presented as numbers and percentages for categorical variables. Medians and ranges for continuous variables are shown as data sets were nonnormally distributed by the Shapiro-Wilk test in the majority of subgroups.

The Hardy-Weinberg equilibrium (HWE) was analyzed to compare the observed genotype frequencies to the expected ones using the Chi-square test $(P>0.01$ with $\mathrm{df}=1$ for equilibrium).

Survival analyses were conducted using the Kaplan-Meier method with the log rank test or with calculation of multiple $P$ value when more than two groups were compared. The Cox proportional hazard model was applied to show whether and to what extend the effect of a unit increase in a covariate was multiplicative with respect to the hazard rate of death. 
TABLE 1: Demographic, clinical, and laboratory characteristics of hemodialysis patients at enrolment into a prospective study $(n=532)$ and their association with all-cause mortality.

\begin{tabular}{|c|c|c|}
\hline Parameter & Value & Association with all-cause mortality ${ }^{\mathrm{a}, \mathrm{b}, \mathrm{c}}$ \\
\hline \multicolumn{3}{|l|}{ Demographic data } \\
\hline Male gender, $n$, $\%$ of all & $297(56.0)$ & 0.360 \\
\hline Age at the beginning of the study, years & $61.2(14.6-89.3)$ & $<0.00001$ \\
\hline RRT vintage prior to the study onset, years & $2.2(0.0-24.7)$ & 0.309 \\
\hline \multicolumn{3}{|l|}{ Cause of ESRD } \\
\hline Diabetic nephropathy, $n$, $\%$ of all & $137(25.8)$ & 0.0002 \\
\hline Chronic glomerulonephritis, $n$, \% of all & $96(18.0)$ & 0.016 \\
\hline Hypertensive nephropathy, $n, \%$ of all & $86(16.2)$ & 0.512 \\
\hline Chronic tubulointerstitial nephritis, $n, \%$ of all & $67(12.6)$ & 0.981 \\
\hline Polycystic kidney disease, $n$, $\%$ of all & $31(5.8)$ & 0.014 \\
\hline Kidney cancer, $n, \%$ of all & $8(1.5)$ & - \\
\hline Urological diseases other than kidney cancer, $n, \%$ of all & $28(5.3)$ & - \\
\hline Myeloma multiplex, $n, \%$ of all & $6(1.1)$ & - \\
\hline Amyloidosis, $n$, \% of all & $7(1.3)$ & - \\
\hline Lupus nephritis, $n, \%$ of all & $2(0.4)$ & - \\
\hline Autoimmune connective tissue disorders other than lupus nephritis, $n, \%$ of all & $9(1.7)$ & - \\
\hline Systemic vasculitis, $n, \%$ of all & $4(0.8)$ & - \\
\hline Rare, $n, \%$ of all & $24(4.5)$ & - \\
\hline Unknown, $n$, \% of all & $27(5.1)$ & - \\
\hline \multicolumn{3}{|l|}{ Clinical data } \\
\hline Coronary artery disease, $n, \%$ of all & $212(39.8)$ & $<0.00001$ \\
\hline Dyslipidemia, $n$, \% of all & $218(41.0)$ & 0.063 \\
\hline History of $\mathrm{HBV}$ infection (anti-HBc positivity), $n, \%$ of all & $128(24.1)$ & 0.303 \\
\hline History of HCV infection (anti-HCV positivity), $n, \%$ of all & $59(11.1)$ & 0.712 \\
\hline HBsAg positivity, $n, \%$ of all & $15(2.8)$ & 0.397 \\
\hline HCV RNA positivity, $n, \%$ of all & $36(6.8)$ & 0.664 \\
\hline Positive anti-HBs, $n, \%$ of all & $457(85.9)$ & 0.013 \\
\hline \multicolumn{3}{|l|}{ Type of RRT } \\
\hline LF-HD, $n, \%$ of all & $277(52.0)$ & 0.853 \\
\hline $\mathrm{HF}-\mathrm{HD}, n, \%$ of all & $217(40.8)$ & 0.390 \\
\hline HDF, $n, \%$ of all & $38(7.1)$ & 0.201 \\
\hline HF-HD/HDF, $n$, $\%$ of all & $255(47.9)$ & 0.853 \\
\hline PD as the first modality of RRT, $n, \%$ of all & $17(3.2)$ & 0.165 \\
\hline \multicolumn{3}{|l|}{ Laboratory data } \\
\hline ALT, IU/L & $13.0(2.0-131.0)$ & 0.863 \\
\hline AST, IU/L & $14.0(3.0-177.0)$ & 0.125 \\
\hline GGT, IU/L & $26.0(1.0-682)$ & 0.089 \\
\hline ALP, U/L & $97.0(38.3-1684)$ & 0.861 \\
\hline PTH, pg/mL & 406 (12.7-3757) & 0.0001 \\
\hline $\mathrm{Ca}, \mathrm{mg} / \mathrm{dL}$ & $8.9(5.4-11.7)$ & 0.089 \\
\hline $\mathrm{P}, \mathrm{mg} / \mathrm{dL}$ & $5.0(2.2-11.3)$ & 0.052 \\
\hline
\end{tabular}

${ }^{\mathrm{a}}$ The Kaplan-Meier analysis was conducted only for uniform parameters exceeding 10 individual reports.

${ }^{\mathrm{b}}$ A multiple $P$ value or a $P$ value calculated using the log rank test, as appropriate.

${ }^{\mathrm{c}}$ Associations were tested in relation to the tertiles of continuous variables.

ALP: alkaline phosphatase, ALT: alanine aminotransferase, Anti-HBc: antibody to core antigen of hepatitis B virus, Anti-HCV: antibody to hepatitis C virus, AST: aspartate aminotransferase, ESRD: end-stage renal disease, GGT: gamma-glutamyl transferase, HBsAg: surface antigen of hepatitis B virus, HBV: hepatitis B virus, HCV: hepatitis C virus, HDF: hemodiafiltration, HF-HD: high flux hemodialysis, LF-HD: low flux hemodialysis, PD: peritoneal dialysis, PTH: parathyroid hormone, RNA: ribonucleic acid, and RRT: renal replacement therapy.

Conversion factors to SI units are as follows: for alanine aminotransferase, $1 \mathrm{U} / \mathrm{L}=0.0167 \mu \mathrm{kat} / \mathrm{L}$, for alkaline phosphatase, $1 \mathrm{U} / \mathrm{L}=0.0167 \mu \mathrm{kat} / \mathrm{L}$, for aspartate aminotransferase, $1 \mathrm{U} / \mathrm{L}=0.0167 \mu \mathrm{kat} / \mathrm{L}$, for calcium, $1 \mathrm{mg} / \mathrm{dL}=0.25 \mathrm{mmol} / \mathrm{L}$, for gamma-glutamyltransferase, $1 \mathrm{U} / \mathrm{L}=0.0167 \mu \mathrm{kat} / \mathrm{L}$, for parathyroid hormone, $1 \mathrm{pg} / \mathrm{mL}=1 \mathrm{ng} / \mathrm{L}$, and for phosphorus, $1 \mathrm{mg} / \mathrm{dL}=0.323 \mathrm{mmol} / \mathrm{L}$. 
TABLE 2: Characteristics of hemodialysis patients at the end of a 7-year prospective study.

\begin{tabular}{lc}
\hline Parameter & Value \\
\hline Demographic data & $7.4(0.5-28.3)$ \\
Total RRT vintage, years & $4.5(0.1-7.0)$ \\
RRT vintage on the prospective study, years & $7(1.3)$ \\
Movement to a noncollaborating dialysis center, $n$, \% of all & $66(12.4)$ \\
Renal transplantation, $n$, \% of all & $317(59.6)$ \\
Causes of death ${ }^{\text {a }}$ & $203(38.2)$ \\
All, $n, \%$ of all & $141(26.5)$ \\
Cardiovascular, $n, \%$ of all & $39(7.3)$ \\
Cardiac, $n, \%$ of all & $30(5.6)$ \\
Sepsis/infection, $n, \%$ of all & $45(8.5)$ \\
Neoplasms, $n, \%$ of all & \\
Rare/unknown, $n, \%$ of all &
\end{tabular}

${ }^{a}$ Death rates are calculated in respect to all patients enrolled in the study $(n=532)$. Outcomes of 66 transplant recipients and 7 patients moving to other centers are not included.

RRT: renal replacement therapy.

TABLE 3: Statistical significance of differences in all-cause mortality evaluated by the Kaplan-Meier analysis for vitamin D signaling pathway genes and CASR in hemodialysis patients undergoing the 7-year prospective study.

\begin{tabular}{|c|c|c|c|c|c|}
\hline $\begin{array}{l}\text { Tested } \\
\text { polymorphism }\end{array}$ & $N$ & $\begin{array}{c}\text { Major homozygotes } \\
\text { versus heterozygotes } \\
\text { versus minor } \\
\text { homozygotes }^{\mathrm{a}} \\
\end{array}$ & $\begin{array}{l}\text { Dominant model of } \\
\text { inheritance }^{b}\end{array}$ & $\begin{array}{l}\text { Recessive model of } \\
\text { inheritance }^{\mathrm{b}}\end{array}$ & $\begin{array}{l}\text { Additive model } \\
\text { of inheritance }^{\text {b }}\end{array}$ \\
\hline GC rs7041 & 458 & $\begin{array}{c}\text { GG versus GT versus } \\
\text { TT } \\
P=0.692\end{array}$ & $\begin{array}{c}\mathrm{TT}+\mathrm{GT} \text { versus } \mathrm{GG} \\
P=0.822\end{array}$ & $\begin{array}{c}\text { TT versus GT + GG } \\
P=0.320\end{array}$ & $\begin{array}{l}\text { TT versus GG } \\
P=0.427\end{array}$ \\
\hline$G C \operatorname{rs} 1155563$ & 472 & $\begin{array}{c}\text { TT versus CT versus } \\
\text { CC } \\
P=0.342\end{array}$ & $\begin{array}{c}\mathrm{CC}+\mathrm{CT} \text { versus } \mathrm{TT} \\
P=0.420\end{array}$ & $\begin{array}{c}\mathrm{CC} \text { versus } \mathrm{CT}+\mathrm{TT} \\
P=0.275\end{array}$ & $\begin{array}{c}\text { CC versus TT } \\
P=0.255\end{array}$ \\
\hline$G C$ rs2298849 & 472 & $\begin{array}{c}\text { TT versus CT versus } \\
\text { CC } \\
P=0.136\end{array}$ & $\begin{array}{c}\mathrm{CC}+\mathrm{CT} \text { versus } \mathrm{TT} \\
\quad P=0.024\end{array}$ & $\begin{array}{c}\mathrm{CC} \text { versus } \mathrm{CT}+\mathrm{TT} \\
P=0.829\end{array}$ & $\begin{array}{c}\text { CC versus TT } \\
P=0.559\end{array}$ \\
\hline $\begin{array}{l}R X R A \\
\text { rs10881578 }\end{array}$ & 472 & $\begin{array}{c}\text { AA versus AG versus } \\
\text { GG } \\
P=0.252\end{array}$ & $\begin{array}{c}\mathrm{GG}+\mathrm{AG} \text { versus } \mathrm{AA} \\
P=0.151\end{array}$ & $\begin{array}{c}\text { GG versus } \mathrm{AG}+\mathrm{AA} \\
P=0.587\end{array}$ & $\begin{array}{l}\text { GG versus AA } \\
P=0.404\end{array}$ \\
\hline $\begin{array}{l}R X R A \\
\text { rs10776909 }\end{array}$ & 472 & $\begin{array}{c}\text { CC versus CT versus } \\
\text { TT } \\
P=0.848\end{array}$ & $\begin{array}{c}\mathrm{TT}+\mathrm{CT} \text { versus } \mathrm{CC} \\
\qquad P=0.672\end{array}$ & $\begin{array}{c}\text { TT versus } \mathrm{CT}+\mathrm{CC} \\
P=0.908\end{array}$ & $\begin{array}{c}\text { TT versus CC } \\
P=0.876\end{array}$ \\
\hline$R X R A$ rs749759 & 464 & $\begin{array}{c}\text { GG versus } A G \text { versus } \\
\text { AA } \\
P=0.516\end{array}$ & $\begin{array}{c}\mathrm{AA}+\mathrm{AG} \text { versus } \mathrm{GG} \\
P=0.263\end{array}$ & $\begin{array}{c}\text { AA versus AG + GG } \\
P=0.942\end{array}$ & $\begin{array}{l}\text { AA versus GG } \\
P=0.830\end{array}$ \\
\hline$V D R$ rs1544410 & 461 & $\begin{array}{c}\text { GG versus } A G \text { versus } \\
\text { AA } \\
P=0.555\end{array}$ & $\begin{array}{c}\mathrm{AA}+\mathrm{AG} \text { versus } \mathrm{GG} \\
P=0.372\end{array}$ & $\begin{array}{c}\text { AA versus AG + GG } \\
\quad P=0.471\end{array}$ & $\begin{array}{c}\text { AA versus GG } \\
P=0.348\end{array}$ \\
\hline$V D R$ rs2228570 & 449 & $\begin{array}{c}\text { CC versus CT versus } \\
\text { TT } \\
P=0.863\end{array}$ & $\begin{array}{c}\mathrm{TT}+\mathrm{CT} \text { versus } \mathrm{CC} \\
\qquad P=0.677\end{array}$ & $\begin{array}{c}\text { TT versus } \mathrm{CT}+\mathrm{CC} \\
P=0.470\end{array}$ & $\begin{array}{c}\text { TT versus CC } \\
P=0.493\end{array}$ \\
\hline CASR rs7652589 & 435 & $\begin{array}{c}\text { GG versus } A G \text { versus } \\
\text { AA } \\
P=0.255\end{array}$ & $\begin{array}{c}\mathrm{AA}+\mathrm{AG} \text { versus } \mathrm{GG} \\
P=0.200\end{array}$ & $\begin{array}{c}\text { AA versus AG + GG } \\
P=0.352\end{array}$ & $\begin{array}{l}\text { AA versus GG } \\
\quad P=0.209\end{array}$ \\
\hline
\end{tabular}

CASR: calcium-sensing receptor gene, GC: group-specific component gene, $R X R A$ : retinoic $\mathrm{X}$ receptor alpha gene, and VDR: vitamin $\mathrm{D}$ receptor gene. ${ }^{a}$ Multiple-sample test $P$.

${ }^{\mathrm{b}}$ Log rank test $P$. 


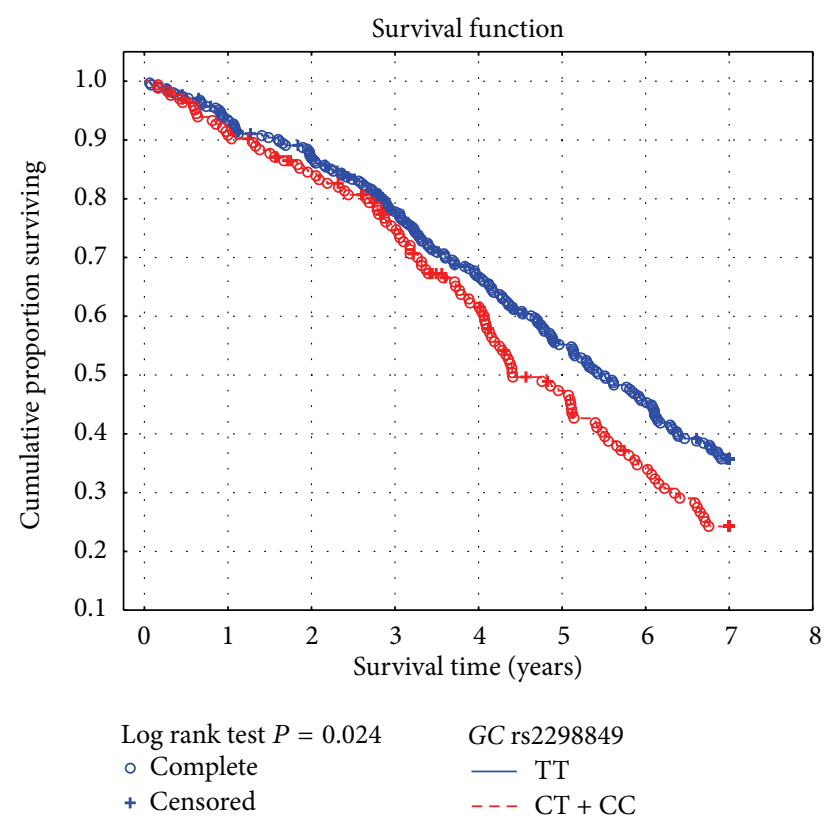

\begin{tabular}{|c|c|c|c|c|c|c|c|c|c|}
\hline \multirow[t]{2}{*}{$\begin{array}{l}G C \text { rs } 2298849 \\
\text { TT versus CT + CC }\end{array}$} & $\begin{array}{l}\text { Parameter } \\
\text { estimate }\end{array}$ & $\begin{array}{l}\text { Standard } \\
\text { error }\end{array}$ & $\begin{array}{l}\chi^{2} \\
\text { test }\end{array}$ & $P$ value & $\begin{array}{c}\text { 95\% lower } \\
\text { CL }\end{array}$ & $\begin{array}{c}\text { 95\% upper } \\
\text { CL }\end{array}$ & $\begin{array}{l}\text { Hazard } \\
\text { ratio }\end{array}$ & $\begin{array}{l}\text { 95\% hazard } \\
\text { ratio lower CL }\end{array}$ & $\begin{array}{l}95 \% \text { hazard } \\
\text { ratio upper CL }\end{array}$ \\
\hline & -0.143 & 0.061 & 5.423 & 0.020 & -0.263 & -0.023 & 0.752 & 0.591 & 0.956 \\
\hline
\end{tabular}

FIGURE 1: The probability of survival in hemodialysis patients in respect to GC rs2298849 polymorphic variant.

Cox proportional hazard model was also applied in multivariate analyses assessing the contribution of demographics and clinical measures to mortality.

Abovementioned statistical analyses were performed using Graph-Pad InStat 3.10, 32 bits for Windows (GraphPad Software, Inc., San Diego, California, United States) and Statistica version 12 (Stat Soft, Inc., Tulsa, Oklahoma, United States).

Haplotype frequencies were estimated using the Haploview 4.2 software (http://www.broad.mit.edu/mpg/ haploview/). Statistical significance was assessed using the 1000 -fold permutation test.

Epistatic interactions were analyzed using the logistic regression and epistasis option in the PLINK software (http:// pngu.mgh.harvard.edu/purcell/plink/).

A $P$ value of less than 0.05 was considered significant. Borderline significance was defined as a $P$ value between 0.05 and 0.10 .

2.5. Ethics Approval of Research. The study design was approved by the Institutional Review Board of Poznan University of Medical Sciences, Poland. The written informed consent was obtained from all study participants.

\section{Results}

At the beginning of the study, distributions of tested polymorphisms were consistent with HWE.
3.1. The Kaplan-Meier Survival Analyses. Association of demographic, clinical, and laboratory characteristics of HD patients at enrolment with their all-cause mortality during the 7-year follow-up is demonstrated in Table 1. Longer survival was attributed to chronic glomerulonephritis (HR $0.675,95 \%$ CI $0.488-0.935, P=0.018$ ) and polycystic kidney disease (HR 0.521, 95\% CI 0.292-0.927, $P=0.027$ ) as causes of ESRD, and the ability to develop antibodies to $\mathrm{HBV}$ surface antigen in response to $\mathrm{HBV}$ vaccination or infection (HR 0.649, 95\% CI 0.478-0.800, $P=0.005$ ). Shorter survival was demonstrated in patients with older age at the beginning of the study (HR 1.027, 95\% CI 1.018-1.035 per each 1-year increase, $P<0.000001$ ), CAD (HR 2.101, 95\% CI 1.682-2.626, $P<0.000001$ ), diabetic nephropathy (HR $1.577,95 \%$ CI 1.248-1.994, $P=0.0001$ ), and lower serum PTH concentrations (HR 1.035, 95\% CI 1.011-1.059 per each $100 \mathrm{pg} / \mathrm{mL}$ decrease, $P=0.004$ ).

Among tested polymorphisms, only GC rs2298849 was significantly associated with all-cause mortality of $\mathrm{HD}$ patients (Table 3). Homozygotes of major allele showed significantly lower risk of death compared to patients bearing the minor allele in GC rs 2298849 (HR 0.75, 95\% CI 0.59-0.96, $P=0.020$, Figure 1). In the next step, we tried to show which of the main causes of death (cardiovascular, infection-related, or neoplasm-related) were the most close to statistical significance being revealed for all-cause mortality. Log rank test, applied as the first-line evaluation, did not show significant $P$ values for cardiovascular (Table 4), infection-related (Supplementary Table 3), and neoplasm-related (Supplementary 
TABLE 4: Statistical significance of differences in cardiovascular mortality evaluated by the Kaplan-Meier analysis for vitamin D signaling pathway genes and CASR in hemodialysis patients undergoing the 7-year prospective study.

\begin{tabular}{|c|c|c|c|c|c|}
\hline Tested polymorphism & $N$ & $\begin{array}{c}\text { Major homozygotes } \\
\text { versus heterozygotes } \\
\text { versus minor } \\
\text { homozygotes }^{\mathrm{a}}\end{array}$ & $\begin{array}{l}\text { Dominant model of } \\
\text { inheritance }^{b}\end{array}$ & $\begin{array}{l}\text { Recessive model of } \\
\text { inheritance }^{b}\end{array}$ & $\begin{array}{l}\text { Additive model } \\
\text { of inheritance }^{b}\end{array}$ \\
\hline$G C$ rs7041 & 458 & $\begin{array}{c}\text { GG versus } \mathrm{GT} \text { versus TT } \\
\qquad P=0.329\end{array}$ & $\begin{array}{c}\mathrm{TT}+\mathrm{GT} \text { versus } \mathrm{GG} \\
P=0.475\end{array}$ & $\begin{array}{c}\text { TT versus GT + GG } \\
P=0.648\end{array}$ & $\begin{array}{c}\text { TT versus GG } \\
P=0.513\end{array}$ \\
\hline$G C$ rs1155563 & 472 & $\begin{array}{c}\text { TT versus CT versus CC } \\
\qquad P=0.316\end{array}$ & $\begin{array}{c}\mathrm{CC}+\mathrm{CT} \text { versus } \mathrm{TT} \\
P=0.542\end{array}$ & $\begin{array}{c}\text { CC versus CT }+\mathrm{TT} \\
P=0.992\end{array}$ & $\begin{array}{l}\text { CC versus TT } \\
P=0.846\end{array}$ \\
\hline GC rs2298849 & 472 & $\begin{array}{c}\text { TT versus CT versus CC } \\
\qquad P=0.906\end{array}$ & $\begin{array}{c}\mathrm{CC}+\mathrm{CT} \text { versus } \mathrm{TT} \\
P=0.797\end{array}$ & $\begin{array}{c}\text { CC versus CT }+\mathrm{TT} \\
P=0.921\end{array}$ & $\begin{array}{c}\text { CC versus TT } \\
P=0.956\end{array}$ \\
\hline$R X R A$ rs10881578 & 472 & $\begin{array}{c}\text { AA versus } A G \text { versus } \\
\text { GG } \\
P=0.778\end{array}$ & $\begin{array}{c}\mathrm{GG}+\mathrm{AG} \text { versus } \mathrm{AA} \\
P=0.617\end{array}$ & $\begin{array}{c}\text { GG versus } \mathrm{AG}+\mathrm{AA} \\
\qquad P=0.391\end{array}$ & $\begin{array}{c}\text { GG versus AA } \\
P=0.516\end{array}$ \\
\hline$R X R A$ rs10776909 & 472 & $\begin{array}{c}\mathrm{CC} \text { versus } \mathrm{CT} \text { versus TT } \\
\qquad P=0.294\end{array}$ & $\begin{array}{c}\mathrm{TT}+\mathrm{CT} \text { versus } \mathrm{CC} \\
P=0.176\end{array}$ & $\begin{array}{c}\text { TT versus CT }+\mathrm{CC} \\
P=0.595\end{array}$ & $\begin{array}{l}\text { TT versus CC } \\
P=0.647\end{array}$ \\
\hline$R X R A$ rs749759 & 464 & $\begin{array}{c}\text { GG versus } A G \text { versus } \\
\begin{array}{c}\text { AA } \\
P=0.705\end{array}\end{array}$ & $\begin{array}{c}\mathrm{AA}+\mathrm{AG} \text { versus } \mathrm{GG} \\
P=0.899\end{array}$ & $\begin{array}{c}\text { AA versus } \mathrm{AG}+\mathrm{GG} \\
\quad P=0.416\end{array}$ & $\begin{array}{l}\text { AA versus GG } \\
\quad P=0.429\end{array}$ \\
\hline$V D R$ rs1544410 & 461 & $\begin{array}{c}\text { GG versus } A G \text { versus } \\
\text { AA } \\
P=0.760\end{array}$ & $\begin{array}{c}\mathrm{AA}+\mathrm{AG} \text { versus } \mathrm{GG} \\
P=0.098\end{array}$ & $\begin{array}{c}\text { AA versus AG + GG } \\
\quad P=0.976\end{array}$ & $\begin{array}{c}\text { AA versus GG } \\
\quad P=0.286\end{array}$ \\
\hline$V D R$ rs2228570 & 449 & $\begin{array}{c}\mathrm{CC} \text { versus } \mathrm{CT} \text { versus TT } \\
\qquad P=0.217\end{array}$ & $\begin{array}{c}\mathrm{TT}+\mathrm{CT} \text { versus CC } \\
P=0.067\end{array}$ & $\begin{array}{c}\text { TT versus CT }+\mathrm{CC} \\
P=0.206\end{array}$ & $\begin{array}{l}\text { TT versus CC } \\
P=0.043\end{array}$ \\
\hline CASR rs7652589 & 435 & $\begin{array}{c}\text { GG versus } A G \text { versus } \\
\text { AA } \\
P=0.119\end{array}$ & $\begin{array}{c}\mathrm{AA}+\mathrm{AG} \text { versus } \mathrm{GG} \\
P=0.781\end{array}$ & $\begin{array}{c}\text { AA versus AG + GG } \\
\quad P=0.090\end{array}$ & $\begin{array}{l}\text { AA versus GG } \\
P=0.168\end{array}$ \\
\hline
\end{tabular}

CASR: calcium-sensing receptor gene, GC: group-specific component gene, RXRA: retinoic X receptor alpha gene, and VDR: vitamin D receptor gene. ${ }^{a}$ Multiple-sample test $P$.

${ }^{\mathrm{b}}$ Log rank test $P$.

Table 4) mortalities in respect to GC rs2298849 polymorphic variants. The HRs with 95\% CI were, respectively, 0.96, 0.71-1.30, $P=0.796$ (Supplementary Figure 1), 0.41, 0.22$0.96, P=0.040$ (Supplementary Figure 2), and 0.64, 0.28$1.47, P=0.291$ (Supplementary Figure 3) for these causes of death analyzed between major allele homozygotes and patients bearing the minor allele. Therefore, the association of GC rs2298849 with cardiovascular mortality could be excluded (Supplementary Figure 1). When noncardiovascular causes of death (infection-related, neoplasm-related, others beyond cardiovascular) were analyzed together in respect to in GC rs2298849 polymorphism, no significance was revealed between major allele homozygotes and patients bearing the minor allele ( $P$ value of 0.252 for $\log$ rank test, HR 0.79 , 95\% CI $0.53-1.18, P=0.243)$. When infection-related and neoplasm-related mortalities were analyzed together, their significant association with GC rs2298849 was shown in the Kaplan-Meier analysis ( $\log$ rank test $P=0.041)$ and in the Cox model (HR 0.53, 95\% CI 0.31-0.92, $P=0.025$ ), indicating better survival in homozygotes of major allele (Figure 2).

Cardiovascular mortality was associated with VDR rs2228570 polymorphism in additive model of inheritance (Table 4). Homozygotes of the major allele showed significantly higher risk of cardiovascular death compared to homozygotes of the minor allele (HR 1.565, 95\% CI 1.0092.426, $P=0.045$, Figure 3 ).
There were no significant associations between infectionrelated (Supplementary Table 3) or neoplasm-related (Supplementary Table 4) mortalities and tested polymorphisms except those already mentioned above for GC rs2298849. $R X R A$ rs10776909 and CASR rs7652589 yielded a borderline significance in respect to infection-related mortality in the dominant model of inheritance (Supplementary Table 3).

3.2. Multivariate Analyses of Survival. Variables that yielded significance in univariate analyses of survival (age at the beginning of the study, diabetic nephropathy, polycystic kidney disease and chronic glomerulonephritis as causes of ESRD, CAD, the ability to develop antibodies to HBV surface antigen in response to $\mathrm{HBV}$ vaccination or infection, and serum PTH concentration) as well as RRT vintage prior to the study onset were used in multivariate analyses together with each polymorphic variant being also solely shown as involved in survival ( $G C$ rs2298849 and VDR rs2228570).

In the Cox model including 8 abovementioned variables and GC rs2298849, significant predictors of 7-year all-cause mortality were age at the beginning of the study (HR 1.016, 95\% CI 1.006-1.026 per each 1-year increase, $P=0.006)$, RRT vintage prior to the study onset (HR 1.055, 95\% CI 1.017-1.094 per each 1-year increase, $P=0.004)$, serum PTH concentration (HR 1.032, 95\% CI 1.004-1.060 per each $100 \mathrm{pg} / \mathrm{mL}$ decrease, $P=0.004$ ), CAD (HR 1.653, 95\% CI 1.285-2.127, 


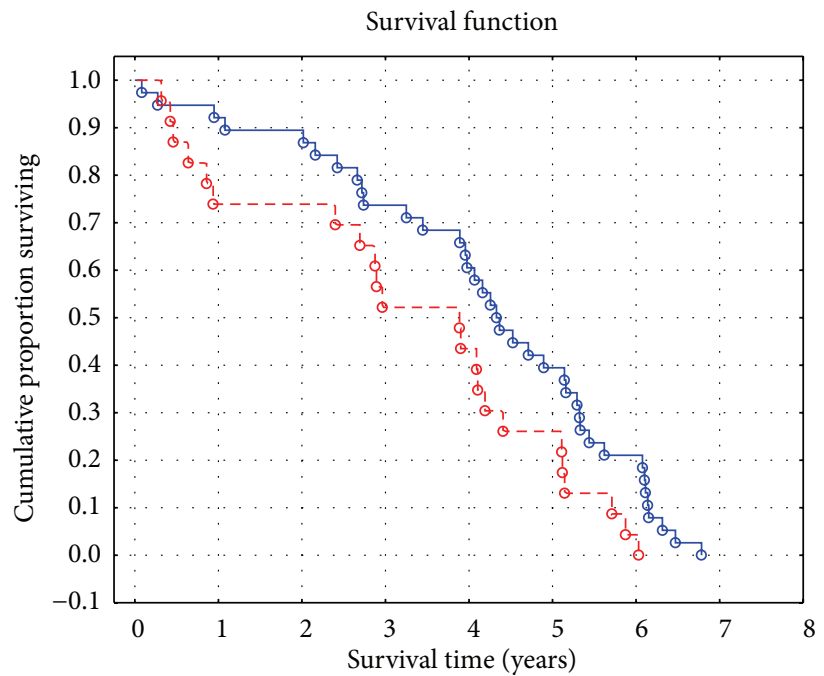

$$
\begin{array}{ll}
\text { Log rank test } P=0.041 & G C \text { rs2298849 } \\
\circ \text { Complete } & - \text { TT } \\
+ \text { Censored } & -- \text { CC + CT }
\end{array}
$$

\begin{tabular}{|c|c|c|c|c|c|c|c|c|c|}
\hline \multirow{2}{*}{$\begin{array}{l}\text { GC rs2298849 } \\
\text { TT versus CC + CT }\end{array}$} & $\begin{array}{l}\text { Parameter } \\
\text { estimate }\end{array}$ & $\begin{array}{l}\text { Standard } \\
\text { error }\end{array}$ & $\begin{array}{c}\chi^{2} \\
\text { test }\end{array}$ & $P$ value & $\begin{array}{c}\text { 95\% lower } \\
\text { CL }\end{array}$ & $\begin{array}{c}95 \% \text { upper } \\
\text { CL }\end{array}$ & $\begin{array}{l}\text { Hazard } \\
\text { ratio }\end{array}$ & $\begin{array}{l}\text { 95\% hazard } \\
\text { ratio lower CL }\end{array}$ & $\begin{array}{l}95 \% \text { hazard } \\
\text { ratio upper CL }\end{array}$ \\
\hline & -0.315 & 0.140 & 5.050 & 0.025 & -0.589 & -0.040 & 0.533 & 0.308 & 0.923 \\
\hline
\end{tabular}

\begin{tabular}{|c|c|c|c|c|c|c|c|c|c|}
\hline \multirow{2}{*}{$\begin{array}{l}V D R \text { rs } 2228570 \\
\text { CC versus TT }\end{array}$} & $\begin{array}{c}\text { Parameter } \\
\text { estimate }\end{array}$ & $\begin{array}{c}\text { Standard } \\
\text { error }\end{array}$ & $\begin{array}{c}\chi^{2} \\
\text { test }\end{array}$ & $P$ value & $\begin{array}{c}95 \% \text { lower } \\
\text { CL }\end{array}$ & $\begin{array}{c}95 \% \text { upper } \\
\text { CL }\end{array}$ & $\begin{array}{c}\text { Hazard } \\
\text { ratio }\end{array}$ & $\begin{array}{l}\text { 95\% hazard } \\
\text { ratio lower CL }\end{array}$ & $\begin{array}{l}95 \% \text { hazard } \\
\text { ratio upper CL }\end{array}$ \\
\hline & 0.224 & 0.112 & 4.006 & 0.045 & 0.005 & 0.443 & 1.565 & 1.009 & 2.426 \\
\hline
\end{tabular}

FIGURE 2: Infection-related and neoplasm-related mortality in hemodialysis patients in respect to GC rs2298849 polymorphic variant.

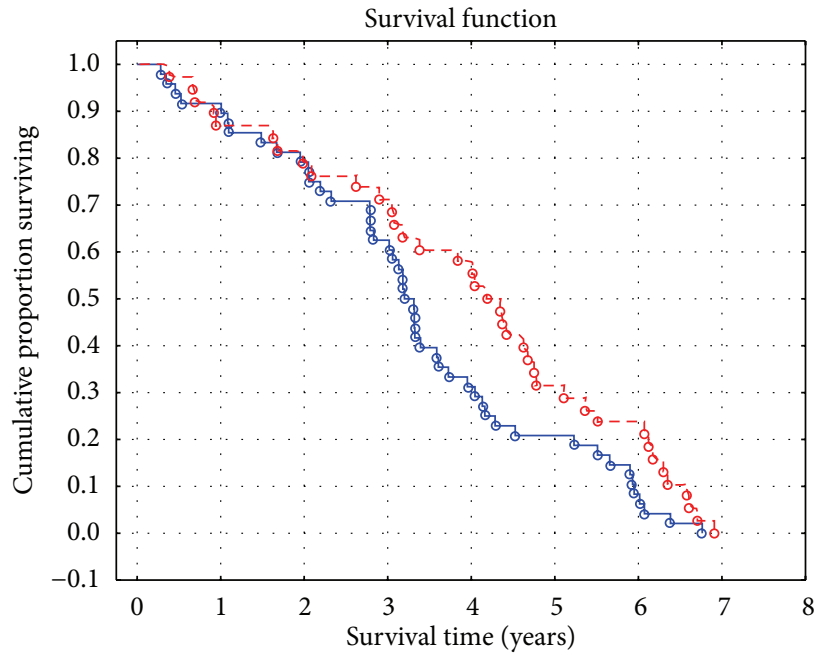

$$
\begin{array}{ll}
\text { Log rank test } P=0.043 & V D R \text { rs } 2228570 \\
\circ \text { Complete } & - \text { CC } \\
+ \text { Censored } & -- \text { TT }
\end{array}
$$

FIGURE 3: Cardiovascular mortality in hemodialysis patients in respect to VDR rs2228570 polymorphic variant. 
$P=0.00009$ ), and the inability to develop antibodies to $\mathrm{HBV}$ surface antigen in response to $\mathrm{HBV}$ vaccination or infection (HR 1.494, 95\% CI 1.090-2.048, $P=0.013$ ). Among these variables, the minor allele in GC rs2298849 showed only borderline association with all-cause mortality since the study beginning (HR 1.253, 95\% CI 0.982-1.599, $P=0.069$ ), maybe due to the fact that patients bearing the minor allele in GC rs2298849 showed longer RRT vintage prior to the study onset compared to homozygotes of the major allele (2.6, 0.0-24.7 years versus $2.0,0.0-22.2$ years, $P=0.023$ ) (Supplementary Table 5). However, the minor allele in $G C$ rs2298849 was found as the significant independent predictor of together analyzed infection-related and neoplasm-related mortalities (HR 2.116, 95\% CI 1.096-4.087, $P=0.026$ ) among the following variables: age at the beginning of the study (HR 0.959, 95\% CI 0.926-0.994 per each 1-year increase, $P=$ 0.021 ), diabetic nephropathy (HR 2.061, 95\% CI 1.012-4.195, $P=0.046$ ), serum PTH concentration (HR 1.088, 95\% CI 1.021-1.159 per each $100 \mathrm{pg} / \mathrm{mL}$ decrease, $P=0.009)$, and the inability to develop antibodies to HBV surface antigen in response to $\mathrm{HBV}$ vaccination or infection (HR 6.204, 95\% CI 2.388-16.119, $P=0.0002$ ).

In multivariate analysis, cardiovascular mortality in $\mathrm{HD}$ patients was associated with CAD (HR 1.671, 95\% CI 1.028$2.718, P=0.038$ ), diabetic nephropathy (HR 1.825, 95\% CI 1.094-3.043, $P=0.021$ ), and major homozygosity (the CC genotype) in VDR rs2228570 (HR 1.896, 95\% CI 1.163-3.091, $P=0.010)$. When death rates due to specific cardiovascular diseases were analyzed in respect to VDR rs2228570 polymorphic variants, the CC genotype was associated with higher risk of sudden cardiac death (OR 2.01, 95\% CI 1.09-3.75, $P=$ $0.024)$. HD patients, who had sudden cardiac death $(n=20)$, showed dyslipidemia in 55\% and CAD in 50\% of cases.

Patients harboring the CC genotype in VDR rs2228570 were more often dyslipidemic compared to subjects showing minor homozygosity (the TT genotype) (46.1\% versus $31.9 \%$, $P=0.047$ ) (Supplementary Table 6). However, dyslipidemia in HD patients was not associated with frequency of major homozygosity in VDR rs2228570. Among all HD subjects tested for VDR rs2228570, dyslipidemic and nondyslipidemic patients did not differ significantly in prevalence of the CC genotype $(31.6 \%$ versus $26.3 \%, P=0.271)$. There was a trend for lower TT genotype frequency in dyslipidemic than in nondyslipidemic patients $(16.0 \%$ versus $24.4 \%, P=$ 0.042). Patients showing the TT genotype were at lower risk of dyslipidemia compared to patients with CC and CT genotypes (HR 0.591, 95\% CI 0.365-0.957, $P=0.042$ ).

3.3. Haplotype Frequencies and Epistatic Interactions. Analyses of haplotype frequencies (Supplementary Tables 7 and 8 ) and epistatic interactions between tested genes (Supplementary Tables 9 and 10) did not reveal significant results in patients who died on HD and those who survived on HD for 7 years as well as in patients who died on HD due to cardiovascular diseases and those who survived on HD for 7 years.

Dyslipidemic HD patients showed higher frequency of the VDR rs1544410_rs2228570 haplotype AC than nondyslipidemic subjects $\left(26 \%\right.$ versus $\left.18 \%, P_{\text {corr }}=0.005\right)$ (Supplementary Table 11). A risk of dyslipidemia was 1.6-fold higher in subjects showing this haplotype (OR 1.640, 95\% CI 1.151-2.338, $P=0.006)$. VDR rs 1544410 and VDR rs 2228570 showed also a significant epistatic interaction (FDR-adjusted $P=0.027$ ) (Supplementary Table 12).

\section{Discussion}

VD deficiency $[3,4]$ and lower plasma concentrations of VD binding protein [16] were already associated with increased mortality risk in HD patients. VDR rs1544410 was shown as influencing survival of Catalonian HD patients in a 4year prospective study [22]. Our 7-year prospective study on Greater Poland HD subjects did not confirm results of this previous study in respect to VDR rs1544410 polymorphism. We have revealed that GC rs2298849 polymorphic variant showed association with together analyzed infectionrelated and neoplasm-related causes mortality, whereas $V D R$ rs2228570 was associated with cardiovascular mortality in prevalence HD patients.

In our retrospective studies, there were no associations between GC rs2298849 and age at RRT onset [32], gender [33], and survival evaluated either since the start of RRT or since birth [2]. GC rs2298849 was also not associated with chronic glomerulonephritis [29] or type 2 diabetic nephropathy [30] as causes of ESRD. CAD in HD patients dialyzed due to type 2 diabetic nephropathy was not found to be associated with $G C$ rs2298849 [30] and a response to $\mathrm{HBV}$ vaccination was not influenced by $G C$ rs2298849 [28]. In this prospective study, the minor allele in GC rs2298849 was recognized as the risk allele for shorter survival on regular HD therapy. The Linkage Disequilibrium (LD) plot of HapMap SNPs within the GC region showed that the rs2298849 variant, located within the second LD block, was in perfect LD $\left(r^{2}=1\right)$ with the intronic variant, rs1352845 [31]. However, this variant was up today associated only with total hip bone mineral density in postmenopausal Thai women [34]. We could not find associations between GC rs2298849 and phenotypes characterizing HD groups categorized by GC rs2298849 genotypes. However, the present study indicates that associations could be searched among infection and neoplasm diseases. The Kaplan-Meier survival curves demonstrate higher infection-cause and neoplasmcause mortalities in HD patients bearing the $\mathrm{C}$ allele in $G C$ rs2298849; however, the independent association of this allele with both mentioned causes of death was not shown in statistical analyses, maybe due to a relatively small number of HD patients dying from infections or neoplasms. As the $\mathrm{C}$ allele in GC rs2298849 seemed to be associated with increased mortality due to infections as well as due to neoplasms, a combined analysis, increasing a number of analyzed patients, yielded statistical significance. Recent data show that GC rs2298849 may be involved in the risk of ovarian cancer among noncarriers of BRCA1/BRCA2 mutations [35]. There is also a possibility that $G C$ rs 2298849 may be associated with infections or neoplasms through its influence on VD status. It is documented that VD deficiency contributes to neoplasm disease [5-7] and infections [36-38]. On the other hand, data on GC rs2298849 in association with VD status are controversial [31, 39-41]. Larger studies evaluating VD 
status, VD associated genes, and specific causes of mortality are needed to show independent associations between tested variables.

GC rs2298849, significantly associated with all-cause mortality in univariate analysis, remained only on borderline level of significance in the Cox multivariate analysis. As our study was performed on prevalent HD patients, bearers of the minor allele in $G C$ rs2298849 appeared to be approximately 6 months longer on RRT prior to the study than homozygotes of the major allele. The study on incidence HD patients could be helpful in avoiding this possible confounding variable.

In Han Chinese adults with normal renal function, the minor allele in VDR rs2228570 was associated with CAD, whereas the major allele homozygosity in VDR rs 2228570 was connected with higher plasma HDL-cholesterol concentrations in CAD patients [42]. Our study showed that the major allele homozygosity in VDR rs2228570 polymorphism was a predictor of cardiovascular mortality in prevalent $H D$ patients. Our study also revealed that VDR rs1544410 and $V D R$ rs2228570 are in epistatic interaction, and the $V D R$ rs1544410_rs2228570 haplotype AC is associated with dyslipidemia in HD patients. Interestingly, López-Mejías et al. [43] recently demonstrated an association of VDR GATG haplotype with atherosclerotic disease in rheumatoid arthritis. The allele T of this haplotype is the minor allele in VDR rs 1544410 and the allele $G$ of this haplotype denotes the major allele in VDR rs2228570. This finding is in logical concordance with our observation showing more dyslipidemic patients among the group presenting the major allele homozygosity in VDR rs2228570 or the VDR rs1544410_rs2228570 haplotype AC. Dyslipidemia, occurring in $46.1 \%$ of the major homozygotes in VDR rs2228570, could contribute to generalized atherosclerosis, CAD, cerebral events, and finally to death. However, death rates due to myocardial infarction or cerebral stroke were not related to the major allele homozygosity in VDR rs2228570. On the other hand, sudden cardiac death, which was shown to be associated with the CC genotype in our study, was also attributed to CAD [44] and abnormal plasma lipids [45], both present in at least $50 \%$ of affected patients.

We were not able to show direct associations of tested polymorphisms with infection-related mortality. However, GC rs2298849, RXRA rs10776909, and CASR rs7652589 yielded a borderline significance in the dominant model of inheritance. In our recent study [24], there was an epistatic interaction between CASR rs7652589 and rs1024611 in the chemokine (C-C motif) ligand 2 gene (CCL2) in nephrolithiasis-related ESRD. The promoter polymorphism of CCL2 was involved in viral [46] and bacterial [47] infections. In this study, 39 patients (7.3\%) died due to infection. Larger group of studied patients or prolongation of the study for further years may clarify this borderline findings.

Neoplasm-related mortality occurred in 5.6\% HD patients and was caused by cancers of various organs (gastrointestinal tract, kidney, bone marrow, and others). This diversity together with low number of affected patients could be a reason why we did not show any associations of tested polymorphisms with a mortality risk due to neoplasms, although such connections were described [35, 48, 49].
Mortality rate in $\mathrm{HD}$ patients in still high. In the study by Buargub [50], 51.4\% patients expired during the 5-year follow-up. In this study, at least $59.6 \%$ patients died during 7 -year period (outcomes of 66 transplant recipients and 7 patients moving to other centers are not included). Therefore, to recognize factors associated with mortality in this group is of great importance. Knowledge of appearance of risk polymorphisms in HD subjects may be helpful in choosing therapeutic strategies. It might be a challenge for the future to find out what outcome after renal transplantation occurs in patients showing the risk polymorphisms in GC rs2298849 or VDR rs2228570.

4.1. Study Limitations. Circulating $25(\mathrm{OH}) \mathrm{D}$ was determined in only 70 patients at the start of the study; therefore sample power was too small for analyses of survival (Supplementary Figure 4). However, determination of total and free vitamin D could be relevant, particularly that serum PTH concentration was among independent predictors of 7-year survival in the examined $\mathrm{HD}$ patients.

\section{Conclusions}

(1) GC rs2298849 polymorphic variant is independently associated with infection/neoplasm mortality, whereas VDR rs2228570 is associated with cardiovascular mortality in prevalence HD patients.

(2) Dyslipidemia occurs more frequently in the major than in the minor homozygotes of VDR rs2228570, and major homozygosity contributes to increased risk of cardiovascular mortality.

(3) The VDR rs1544410_rs2228570 haplotype AC is associated with dyslipidemia in HD patients, whereas the TT genotype in VDR rs2228570 seems to have a protective role against dyslipidemia.

\section{Competing Interests}

The authors declare that there is no conflict of interests regarding the publication of this paper.

\section{Acknowledgments}

The authors would like to express our gratitude to the physicians of the dialysis centers for their consent in collecting the participants' data. This study was funded in part by the scientific grant of the Baxter company allocated by the Chapter of the Polish Society of Nephrology as the blindly reviewed award winning project, Grant no. 504-04-02225363-0001303071. Additional funding was provided by the Poznan University of Medical Sciences, Poznań, Poland, Grant nos. 502-01-02225363-03679 and 502-01-01124182-07474.

\section{References}

[1] United States Renal Data System, 2015 USRDS Annual Data Report: Epidemiology of Kidney Disease in the United States, National Institutes of Health, National Institute of Diabetes and Digestive and Kidney Diseases, Bethesda, Md, USA, 2015. 
[2] M. Świderska, A. Mostowska, and A. E. Grzegorzewska, "T helper cell related cytokine gene polymorphisms and vitamin D pathway gene polymorphisms as predictors of survival probability in patients on renal replacement therapy," Polskie Archiwum Medycyny Wewnętrznej, vol. 125, pp. 511-520, 2015.

[3] G. Jean, B. Charra, and C. Chazot, "Vitamin D deficiency and associated factors in hemodialysis patients," Journal of Renal Nutrition, vol. 18, no. 5, pp. 395-399, 2008.

[4] A. Schiller, F. Gadalean, O. Schiller et al., "Vitamin D deficiency-prognostic marker or mortality risk factor in end stage renal disease patients with diabetes mellitus treated with hemodialysis-a prospective multicenter study," PLOS ONE, vol. 10, no. 5, Article ID e0126586, 2015.

[5] C. J. Narvaez, D. Matthews, E. LaPorta, K. M. Simmons, S. Beaudin, and J. Welsh, "The impact of vitamin D in breast cancer: genomics, pathways, metabolism," Frontiers in Physiology, vol. 5, article 213, pp. 158-167, 2014.

[6] V. Fedirko, E. Riboli, A. Tjønneland et al., "Prediagnostic 25-hydroxyvitamin D, VDR and CASR polymorphisms, and survival in patients with colorectal cancer in western european populations," Cancer Epidemiology Biomarkers and Prevention, vol. 21, no. 4, pp. 582-593, 2012.

[7] S. Meeker, A. Seamons, J. Paik et al., "Increased dietary vitamin D suppresses MAPK signaling, colitis, and colon cancer," Cancer Research, vol. 74, no. 16, pp. 4398-4408, 2014.

[8] E. Hyppönen, E. Läärä, A. Reunanen, M.-R. Järvelin, and S. $\mathrm{M}$. Virtanen, "Intake of vitamin $\mathrm{D}$ and risk of type 1 diabetes: a birth-cohort study," The Lancet, vol. 358, no. 9292, pp. 15001503, 2001.

[9] U. Kampmann, L. Mosekilde, C. Juhl et al., "Effects of 12 weeks high dose vitamin D3 treatment on insulin sensitivity, beta cell function, and metabolic markers in patients with type 2 diabetes and vitamin D insufficiency-a double-blind, randomized, placebo-controlled trial," Metabolism: Clinical and Experimental, vol. 63, no. 9, pp. 1115-1124, 2014.

[10] J. A. Ford, G. S. MacLennan, A. Avenell, M. Bolland, A. Grey, and M. Witham, "Cardiovascular disease and vitamin D supplementation: trial analysis, systematic review, and metaanalysis," The American Journal of Clinical Nutrition, vol. 100, no. 3, pp. 746-755, 2014.

[11] M. Karakas, B. Thorand, A. Zierer et al., "Low levels of serum 25-hydroxyvitamin D are associated with increased risk of myocardial infarction, especially in women: results from the MONICA/KORA Augsburg case-cohort study," Journal of Clinical Endocrinology and Metabolism, vol. 98, no. 1, pp. 272280, 2013.

[12] Y. Sato, T. Asoh, I. Kondo, and K. Satoh, "Vitamin D deficiency and risk of hip fractures among disabled elderly stroke patients," Stroke, vol. 32, no. 7, pp. 1673-1677, 2001.

[13] H. Wen and J. F. Baker, "Vitamin D, immunoregulation, and rheumatoid arthritis," Journal of Clinical Rheumatology, vol. 17, no. 2, pp. 102-107, 2011.

[14] L. L. Ritterhouse, S. R. Crowe, T. B. Niewold et al., "Vitamin D deficiency is associated with an increased autoimmune response in healthy individuals and in patients with systemic lupus erythematosus," Annals of the Rheumatic Diseases, vol. 70, no. 9, pp. 1569-1574, 2011.

[15] O. Evliyaoğlu, M. Acar, B. Özcabı et al., "Vitamin D defciency and hashimoto's thyroiditis in children and adolescents: a critical vitamin D level for this association?" Journal of Clinical Research in Pediatric Endocrinology, vol. 7, no. 2, pp. 128-133, 2015.
[16] Y.-P. Lin, C.-Y. Yang, C.-C. Liao, W.-C. Yu, C.-W. Chi, and C.-H. Lin, "Plasma protein characteristics of long-term hemodialysis survivors," PLoS ONE, vol. 7, no. 7, Article ID e40232, 2012.

[17] B. R. Santos, L. P. G. Mascarenhas, F. Satler, M. C. S. Boguszewski, and P. M. Spritzer, "Vitamin D deficiency in girls from South Brazil: a cross-sectional study on prevalence and association with vitamin D receptor gene variants," $B M C$ Pediatrics, vol. 12, article 62, 2012.

[18] E. A. Hibler, P. W. Jurutka, J. B. Egan et al., "Association between polymorphic variation in VDR and RXRA and circulating levels of vitamin D metabolites," Journal of Steroid Biochemistry and Molecular Biology, vol. 121, no. 1-2, pp. 438-441, 2010.

[19] K. Batai, A. B. Murphy, E. Shah et al., "Common vitamin D pathway gene variants reveal contrasting effects on serum vitamin $D$ levels in African Americans and European Americans," Human Genetics, vol. 133, no. 11, pp. 1395-1405, 2014.

[20] S. Smajilovic, S. Yano, R. Jabbari, and J. Tfelt-Hansen, "The calcium-sensing receptor and calcimimetics in blood pressure modulation," British Journal of Pharmacology, vol. 164, no. 3, pp. 884-893, 2011.

[21] M. Tokumoto, M. Taniguchi, D. Matsuo, K. Tsuruya, H. Hirakata, and M. Iida, "Parathyroid cell growth in patients with advanced secondary hyperparathyroidism: vitamin D receptor, calcium sensing receptor, and cell cycle regulating factors," Therapeutic Apheresis and Dialysis, vol. 9, supplement 1, pp. S27S34, 2005.

[22] M. P. Marco, L. Craver, A. Betriu, J. Fibla, and E. Fernández, "Influence of vitamin D receptor gene polymorphisms on mortality risk in hemodialysis patients," American Journal of Kidney Diseases, vol. 38, no. 5, pp. 965-974, 2001.

[23] L. Lai, J. Qian, Y. Yang et al., "Is the serum vitamin D level at the time of hospital-acquired acute kidney injury diagnosis associated with prognosis?" PLoS ONE, vol. 8, no. 5, Article ID e64964, 2013.

[24] A. Grzegorzewska, M. Paciorkowski, A. Mostowska et al., "Associations of single nucleotide polymorphism of the calciumsensing receptor gene (CASR rs7652589) with nephrolithiasis and secondary hyperparathyroidism in hemodialysis patients," in Proceedings of the 53rd ERA-EDTA Congress, Vienna, Austria, May 2016.

[25] V. N. Babinsky, F. M. Hannan, S. C. Youhanna et al., "Association studies of calcium-sensing receptor (C $\alpha \mathrm{SR})$ polymorphisms with serum concentrations of glucose and phosphate, and vascular calcification in renal transplant recipients," PLoS ONE, vol. 10, no. 3, Article ID e0119459, 2015.

[26] National Kidney Foundation and Kidney Disease Outcomes Quality Initiative (K/DOQI) Group, "K/DOQI clinical practice guidelines for management of dyslipidemias in patients with dialysis-dependent chronic kidney disease," American Journal of Kidney Diseases, vol. 41, supplement 3, pp. S3-S91, 2003.

[27] A. E. Grzegorzewska, M. K. Świderska, and W. Warchoł, "Antibodies to hepatitis B virus surface antigen and survival of hemodialysis patients-a prospective study," Expert Review of Vaccines, vol. 15, no. 8, pp. 1063-1074, 2016.

[28] A. E. Grzegorzewska, E. Jodłowska, A. Mostowska, A. Sowińska, and P. P. Jagodziński, "Single nucleotide polymorphisms of vitamin $\mathrm{D}$ binding protein, vitamin $\mathrm{D}$ receptor and retinoid $\mathrm{X}$ receptor alpha genes and response to hepatitis $\mathrm{B}$ vaccination in renal replacement therapy patients," Expert Review of Vaccines, vol. 13, no. 11, pp. 1395-1403, 2014.

[29] A. E. Grzegorzewska, G. Ostromecki, P. Zielińska et al., “Association of retinoid $\mathrm{X}$ receptor alpha gene polymorphism with 
clinical course of chronic glomerulonephritis," Medical Science Monitor, vol. 21, article A497, pp. 3671-3681, 2015.

[30] A. E. Grzegorzewska, G. Ostromecki, P. Zielińska, A. Mostowska, and P. P. Jagodziński, "T-Cell cytokine gene polymorphisms and vitamin $\mathrm{D}$ pathway gene polymorphisms in end-stage renal disease due to type 2 diabetes mellitus nephropathy: comparisons with health status and other main causes of end-stage renal disease," Journal of Diabetes Research, vol. 2014, Article ID 120317, 17 pages, 2014.

[31] A. E. Grzegorzewska, G. Ostromecki, A. Mostowska, A. Sowiñska, and P. P. Jagodziñski, "Clinical aspects of vitamin Dbinding protein gene polymorphisms in hemodialysis patients," Polskie Archiwum Medycyny Wewnetrznej, vol. 125, no. 1-2, pp. 8-17, 2015.

[32] A. E. Grzegorzewska, G. Ostromecki, A. Mostowska et al., "Vitamin D pathway genes in relation to age at renal replacement therapy onset," in Proceedings of the ASN Kidney Week, Philadelphia, Pa, USA, November 2014, Journal of the American Society of Nephrology, vol. 25, abstract supplement, 565A, 2014.

[33] A. E. Grzegorzewska, G. Ostromecki, A. Mostowska et al., "Clinical aspects of vitamin D pathway gene polymorphism in hemodialysis women and men," in Proceedings of the 51st ERAEDTA Congress, Amsterdam, The Netherlands, May-June 2014, Nephrology Dialysis Transplantation, vol. 29, supplement 3, pp. iii505-iii506, 2014.

[34] C. Chupeerach, A. Tungtrongchitr, B. Phonrat, F. J. Schweigert, R. Tungtrongchitr, and S. Preutthipan, "Association of Thr420Lys polymorphism in DBP gene with fat-soluble vitamins and low radial bone mineral density in postmenopausal Thai women," Biomarkers in Medicine, vol. 6, no. 1, pp. 103-108, 2012.

[35] A. Mostowska, S. Sajdak, P. Pawlik, M. Lianeri, and P. P. Jagodzinski, "Polymorphic variants in the vitamin D pathway genes and the risk of ovarian cancer among non-carriers of BRCA1/BRCA2 mutations," Oncology Letters, vol. 11, no. 2, pp. 1181-1188, 2016.

[36] J. Yang, G. Chen, D. Wang, M. Chen, C. Xing, and B. Wang, "Low serum 25-hydroxyvitamin D level and risk of urinary tract infection in infants," Medicine, vol. 95, no. 27, Article ID e4137, 2016.

[37] M. Haliloglu, B. Bilgili, O. Haliloglu, D. Gogas Yavuz, and I. Cinel, "Vitamin D level is associated with mortality predictors in ventilator-associated pneumonia caused by Acinetobacter baumannii," The Journal of Infection in Developing Countries, vol. 10, no. 6, pp. 567-574, 2016.

[38] A. Zittermann, J. Kuhn, J. B. Ernst et al., "Circulating 25hydroxyvitamin D and 1,25-dihydroxyvitamin D Concentrations and postoperative infections in cardiac surgical patients: the CALCITOP-study," PLoS ONE, vol. 11, no. 6, Article ID e0158532, 2016.

[39] J. Ahn, K. Yu, R. Stolzenberg-Solomon et al., "Genome-wide association study of circulating vitamin D levels," Human Molecular Genetics, vol. 19, no. 13, pp. 2739-2745, 2010.

[40] W. Xu, J. Sun, W. Wang et al., "Association of genetic variants of vit D binding protein (DBP/GC) and of the enzyme catalyzing its 25-hydroxylation (DCYP2R1) and serum vit D in postmenopausal women," Hormones, vol. 13, no. 3, pp. 345-352, 2014.

[41] L. Foucan, F.-L. Vélayoudom-Céphise, L. Larifla et al., "Polymorphisms in GC and NADSYN1 Genes are associated with vitamin D status and metabolic profile in Non-diabetic adults," BMC Endocrine Disorders, vol. 13, article 36, 2013.
[42] L. He and M. Wang, "Association of vitamin D receptor-a gene polymorphisms with coronary heart disease in Han Chinese," International Journal of Clinical and Experimental Medicine, vol. 8, no. 4, pp. 6224-6229, 2015.

[43] R. López-Mejías, F. Genre, S. Remuzgo-Martínez et al., "Vitamin D receptor GATG haplotype association with atherosclerotic disease in patients with rheumatoid arthritis," Atherosclerosis, vol. 245, pp. 139-142, 2016.

[44] R. J. Myerburg and M. J. Junttila, "Sudden cardiac death caused by coronary heart disease," Circulation, vol. 125, no. 8, pp. 10431052, 2012.

[45] A. Tsuji, N. Ikeda, and T. Nakamura, "Plasma lipids, lipoproteins and apolipoproteins and sudden cardiac death," International Journal of Legal Medicine, vol. 112, no. 3, pp. 151-154, 1999.

[46] L. P. Byung, J. K. Yoon, S. C. Hyun et al., "Association of common promoter polymorphisms of MCP1 with hepatitis B virus clearance," Experimental and Molecular Medicine, vol. 38, no. 6, pp. 694-702, 2006.

[47] Y. Zhang, J. Zhang, L. Zeng et al., "The -2518A/G polymorphism in the MCP-1 gene and tuberculosis risk: a meta-analysis," PLoS ONE, vol. 7, no. 7, Article ID e38918, 2012.

[48] W. Maneechay, T. Boonpipattanapong, S. Kanngurn, P. Puttawibul, S. L. Geater, and S. Sangkhathat, "Single nucleotide polymorphisms in the Gc gene for vitamin D binding protein in common cancers in Thailand," Asian Pacific Journal of Cancer Prevention, vol. 16, no. 8, pp. 3339-3344, 2015.

[49] S. Jeong, J. H. Kim, M. G. Kim et al., "Genetic polymorphisms of CASR and cancer risk: evidence from meta-analysis and $\mathrm{HuGe}$ review," OncoTargets and Therapy, vol. 9, pp. 655-669, 2016.

[50] M. A. Buargub, "5-Year mortality in hemodialysis patients: a single center study in Tripoli," Saudi Journal of Kidney Diseases and Transplantation, vol. 19, no. 2, pp. 268-273, 2008. 


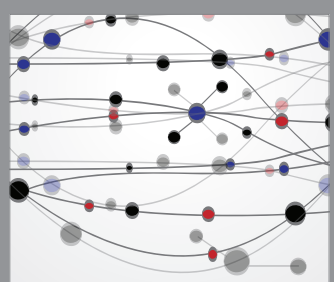

The Scientific World Journal
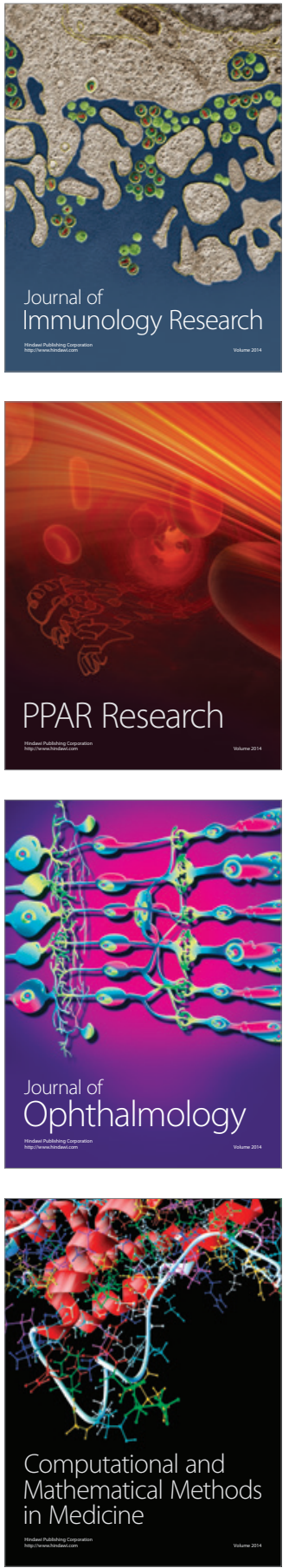

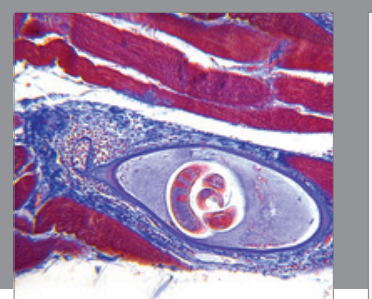

Gastroenterology Research and Practice

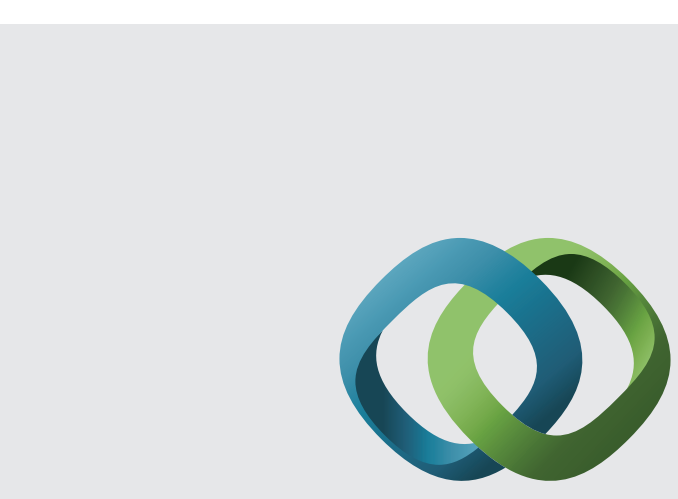

\section{Hindawi}

Submit your manuscripts at

http://www.hindawi.com
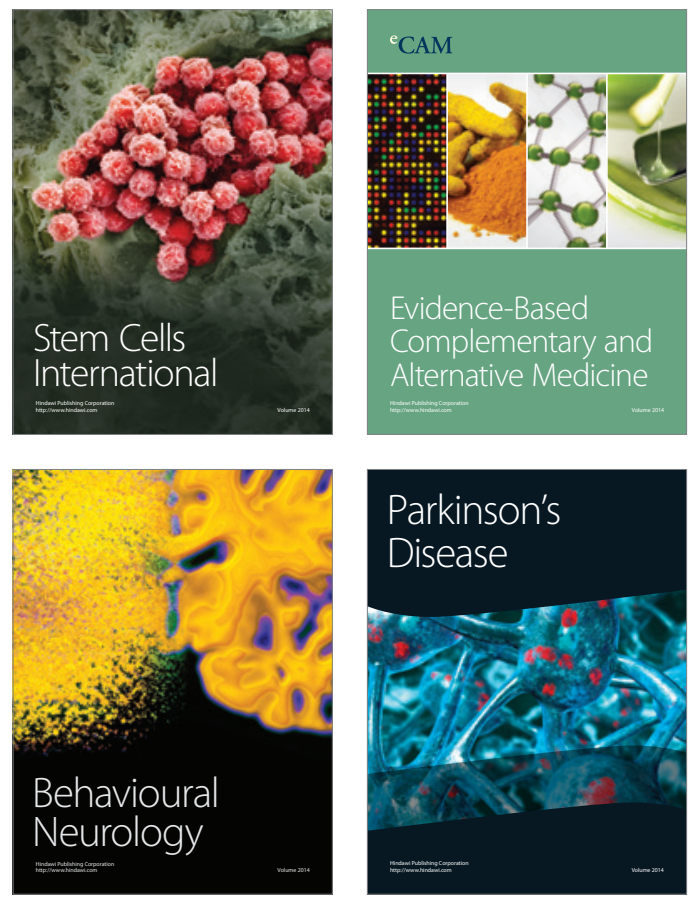
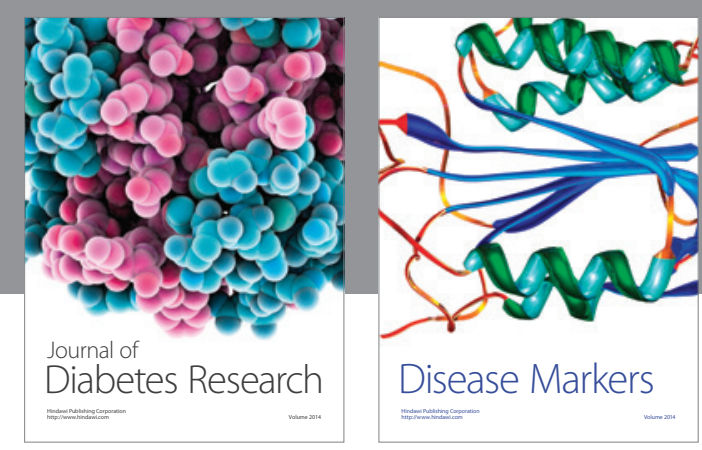

Disease Markers
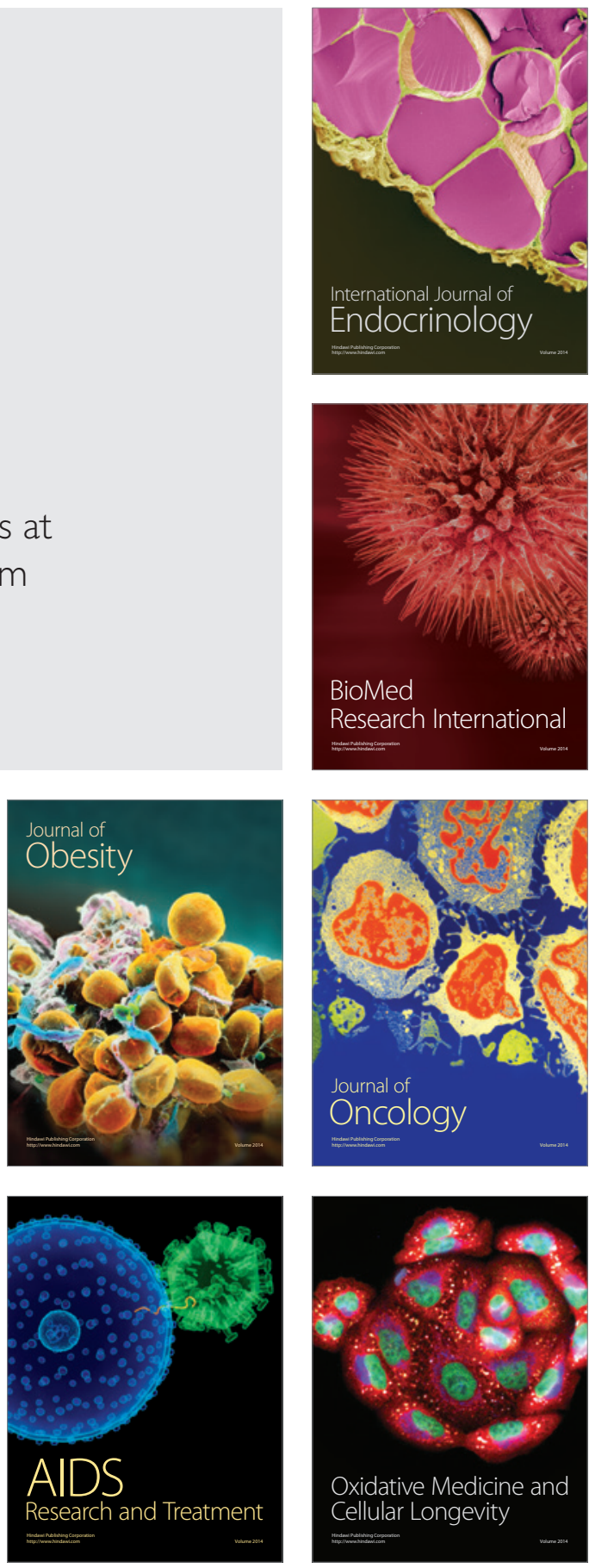